\title{
X-MCD STUDY OF EXPANDED LATTICE PERMANENT MAGNET MATERIALS
}

\author{
Cz. Kapusta, R. Mycielski, B. Porębska \\ Department of Solid State Physics, University of Mining and Metallurgy \\ Al. Mickiewicza 30, 30-059 Cracow, Poland \\ D. Ahlers, K. Attenkofer, P. Fischer and G. Schütz
}

Institute of Experimental Physics II, University of Augsburg, 86135 Augsburg, Germany

X-ray magnetic circular dichroism measurements on the cobalt (iron) $K$ edges and samarium $L_{2}, L_{3}$ edges of $\mathrm{Sm}_{2} \mathrm{Fe}_{17}, \mathrm{Sm}_{2} \mathrm{Co}_{17}$ and their interstitial hydrides and nitrides are reported. Normal absorption profiles show considerable changes upon nitriding for iron- and cobalt $K$ edge and for samarium $L_{2}, L_{3}$ edges. Much weaker changes are observed upon hydriding. Similarly, significant differences of X-MCD spectra appear between the host $\mathrm{Sm}_{2} \mathrm{Fe}_{17}\left(\mathrm{Sm}_{2} \mathrm{Co}_{17}\right)$ and the nitrides, whereas $\mathrm{X}-\mathrm{MCD}$ signals of the hydrides are similar to those of the hosts. The effects are analysed and discussed in terms of influence of interstitial atom neighbours on the $\mathrm{Co}(\mathrm{Fe}) 4 p$ and Sm $5 d$ electronic bands.

PACS numbers: $75.30 .-\mathrm{m}, 78.70 . \mathrm{Dm}$

\section{Introduction}

The compounds belong to the family of materials for high performance permanent magnets deriving from $\mathrm{Sm}_{2} \mathrm{Co}_{17}$. The $\mathrm{Fe}$ based compound, $\mathrm{Sm}_{2} \mathrm{Fe}_{17}$, however, having comparatively low magnetic ordering temperature and a planar anisotropy has not been a candidate for applications until recently, when it was found that its properties could be dramatically improved by introduction of carbon or nitrogen on the interstitial sites in the structure [1,2]. A huge rise of magnetic ordering temperature and appearance of a strong uniaxial anisotropy is observed upon nitriding or carbiding. A smaller effect of hydrogen on Curie temperature, $T_{\mathrm{C}}$, is observed and a planar anisotropy of the host $\mathrm{Sm}_{2} \mathrm{Fe}_{17}$ material persists in the hydride. The change of $T_{\mathrm{C}}$ is caused by the magnetovolume effect of nitriding (hydriding) which acts towards localization of the Fe $3 d$ electronic band. Appearance of a huge uniaxial anisotropy in the nitride is related with nitrogen atoms neighbouring the $\mathrm{Sm}$ atoms in the structure and changing the distribution of the $\mathrm{Sm} 5 d$ and $6 p$ valence electrons [3]. Changes of the Sm valence electron polarization caused by interstitial hydrogen, nitrogen and carbon were found to be reflected 
in the electric field gradient (EFG) and magnetic hyperfine field (IIFF) observed recently in samarium nuclear magnetic resonance (NMR) measurements $[4,5]$. In order of deeper insight into the influence of interstitial hydrogen and nitrogen atoms on the $\mathrm{Sm} 5 d$ and the $\mathrm{Co}(\mathrm{Fe}) 4 p$ electronic bands and their polarization the present X-MCD and X-ray absorption near-edge structure (XANES) study was undertaken.

\section{Experimental}

The measurements were carried out using the inclined view, two beam method at the RÖMO II station at HASYLAB/DESY in Hamburg [6]. Polycrystalline samples of $\mathrm{Sm}_{2} \mathrm{Co}_{17}, \mathrm{Sm}_{2} \mathrm{Co}_{17} \mathrm{~N}_{3}, \quad \mathrm{Sm}_{2} \mathrm{Co}_{17} \mathrm{H}_{4.6}, \mathrm{Sm}_{2} \mathrm{Fe}_{17}, \quad \mathrm{Sm}_{2} \mathrm{Fe}_{17} \mathrm{~N}_{2.7}$ and $\mathrm{Sm}_{2} \mathrm{Fe}_{17} \mathrm{H}_{2.9}$ were used. Each sample had a form of two thin layers of fine powder spread on a transparent adhesive tape. The sample was mounted inside a water cooled solenoid and the measurements were carried out at room temperature. The X-MCD spectra presented are extrapolated to a $100 \%$ degree of circular polarization. The zero point is set to the first inflection point of the absorption onset coinciding roughly with the Fermi level. At the $K$ edges one generally speaks of the pre-peak structure.

\section{Results and discussion}

Normal absorption profiles at the $\mathrm{Co}(\mathrm{Fe})$ edges (Fig. 1) show a smoothing of the middle-edge kink for the nitrides, whereas for hydrides the changes are much smaller compared to the host compound. The effect is attributed to a strong influence of neighbouring nitrogen atoms on the density of $4 p$ states being the final state for the $K$ edge transitions. The effect of hydrogen atom neighbours on the $\mathrm{Co}(\mathrm{Fe}) 4 p$ states is found to be much weaker.
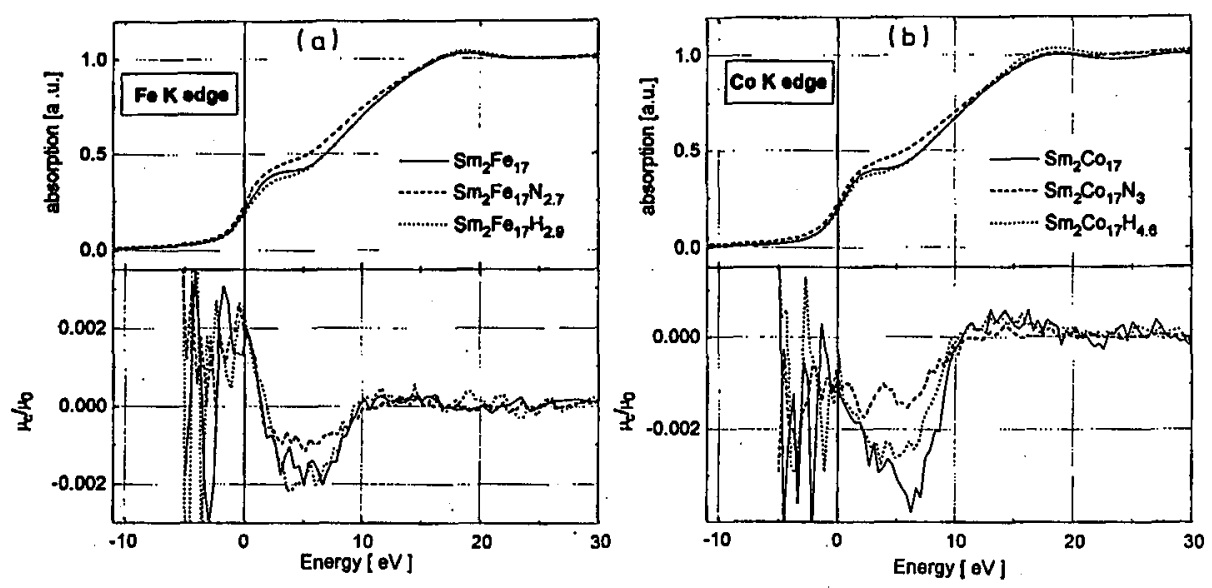

Fig. 1. Normal absorption and dichroic signal at (A) Fe $K$ edge and (B) Co $K$ edge in different samples (as indicated). 


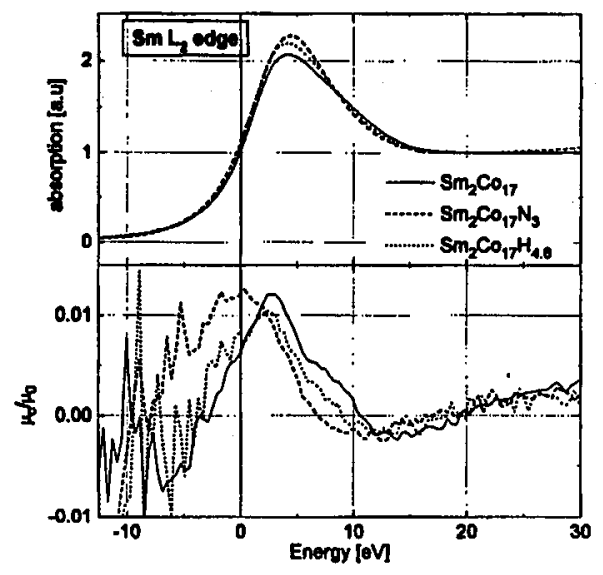

Fig. 2. Normal absorption and dichroic signal at $\operatorname{Sm} L_{2}$ edge in different samples (as indicated).
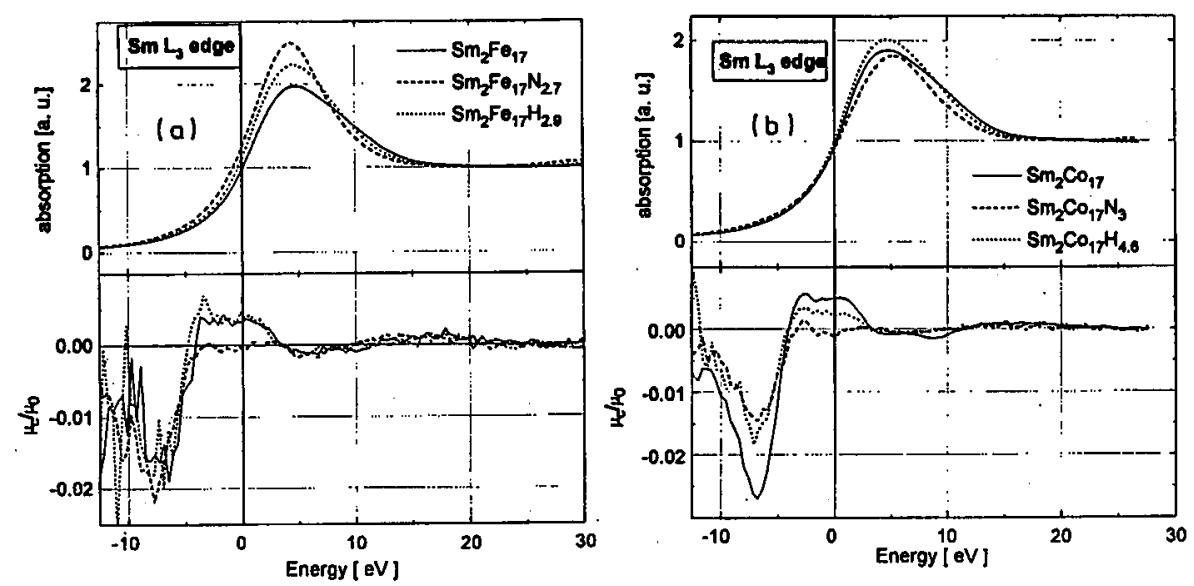

Fig. 3. Normal absorption and dichroic signal at $\mathrm{Sm} L_{3}$ edges in different samples (as indicated).

The "white line" at the $L_{2}, L_{3}$ edges (Figs. 2,3) reveals a narrowing upon interstitial modification. The effect is much stronger for the nitrides than for the hydrides, which we attribute to a larger narrowing and stronger localization of the Sm $5 d$ band under influence of nitrogen neighbours than that caused by hydrogen.

The X-MCD signals at Fe $K$ edges (Fig. 1) show a double peak structure consisting of a negative peak at $E>0$ and a positive one around zero energies. The structure indicates that the $\mathrm{Fe} 4 p$ band resembles a weak band ferromagnetism of iron with the incompletely filled $3 d$ majority band [7]. Upon hydriding no significant change of the signal is observed, whereas nitriding leads to a decrease in intensity of the negative signal at $E>0$. From this result an impact of nitrogen neighbours on the $\mathrm{Fe} 4 p$ band polarization can be deduced, whereas the influence 
of hydrogen is negligible. Persistence of the positive signal at zero energies in the nitride and hydride allows to conclude that interstitial modification does not lead to a strong band ferromagnetism of iron.

A single negative peak is observed in the Co $K$ edge X-MCD spectra, reflecting strong band ferromagnetism of cobalt in the compounds. Similarly to the Fe based compounds, nitrogen causes a strong reduction of the intensity of the peak. Some effect of hydrogen on the peak shape can possibly be related with its higher content than for the Fe based compounds, and thus, with occupation of tetrahedral sites besides octahedral sites, which occurs for hydrogen content larger than 3 atoms/f.u. [8].

The $\mathrm{Sm} L_{3}$ edge X-MCD spectra (Fig. 3) show a disappearance of the positive signal around zero energies for the nitrides, whereas it persists for the hydrides. Following [9] the influence is attributed to a decrease in $5 d-3 d$ overlap caused by covalent effect of nitrogen, whereas hydrogen has a negligible impact on it. The effect is consistent with a significant decrease in the molecular field coefficient $n_{\mathrm{RT}}$ found in magnetic and neutron measurements for the nitrides [10]. The decrease in the positive signal can also be interpreted in a simple vector coupling model to result from a decreased orbital contribution, which in turn is related to a huge rise of magnetocrystalline anisotropy with nitriding. The prominent negative signal at $E<0$ remains unchanged, indicating its localised, atomic character.

The X-MCD signals at the $L_{2}$ edge of $\mathrm{Sm}$ in the Co-based nitride (Fig. 2) show a broadening and a shift of the main peak to $0 \mathrm{eV}$ from $3 \mathrm{eV}$ in the host $\mathrm{Sm}_{2} \mathrm{Co}_{17}$, as well as a decrease in the kink at $8 \mathrm{eV}$. Minor changes are observed upon hydriding. Since the $L_{2}$ edge is sensitive to the chemical environment [9], the effect, together with the $L_{3}$ signal changes indicates much stronger influence of covalent effects on the Sm $5 d$ band for nitrogen than for hydrogen. The result is consistent with the Sm NMR measurements, where a strong effect of nitrogen and no effect of hydrogen on the $5 d(6 p)$ bands was observed in the electric field gradient and magnetic hyperfine field.

\section{Conclusions}

Interstitial nitrogen atoms have a considerable impact on the rare earth $5 d$ and the $\mathrm{Co}(\mathrm{Fe}) 4 p$ electronic bands. It involves a narrowing of the $5 d$ band and a decrease in $5 d-3 d$ overlap and, thus, a decrease in its polarization by neighbouring $\mathrm{Co}(\mathrm{Fe})$ spins. Also, the polarization of the $\mathrm{Co}(\mathrm{Fe}) 4 p$ band is influenced. Much less pronounced effects of hydrogen are observed.

Upon hydriding or nitriding the iron-based compounds remain weak band ferromagnets with incompletely filled $3 d$ minority band, whereas the cobalt-based compounds keep strong Co $3 d$ band ferromagnetism.

Work supported by the Committee for Scientific Research (Poland), project no. 2P03B 11909 and BMFT (Germany), project no. 05 5WOEAI 8.

\section{References}

[1] D.B. de Mooij, K.H.J. Buschow, J. Less-Common. Met. 142, 349 (1988).

[2] Hong Sun, J.M.D. Coey, Y. Otani, D.P.F. Hurley, J. Phys., Condens. Matter 2, 6465 (1990). 
[3] R. Coehoorn, K.H.J. Buschow, J. Appl. Phys. 69, 5590 (1991).

[4] Cz. Kapusta, P.C. Riedi, in: NATO ASI Series, Vol. 281, Eds. F. Grandjean, G.J. Long, K.H.J. Buschow, Kluwer, Dordrecht 1995, p. 497.

[5] Cz. Kapusta, P.C. Riedi, J.S. Lord, R. Mycielski, K.H.J. Buschow, J. Alloys Comp. 235, 66 (1996).

[6] G. Schütz, R. Frahm, R. Wienke, W. Wilhelm, W. Wagner, P. Kienle, Rev. Sci. Instrum. 60, 1661 (1989).

[7] S. Stähler, G. Schütz, H. Ebert, Phys. Rev. B 47, 818 (1993).

[8] O. Isnard, S. Miraglia, J.L. Soubeyroux, D. Fruchart, Solid State Commun. 81, 13 (1992).

[9] P. Fischer, Ph.D. Thesis, Technical University Munich, 1993.

[10] M. Löwenhaupt, P. Tils, D.P. Middleton, K.H.J. Buschow, R. Eccleston, J. Magn. Magn. Mater. 129, L151 (1994). 